complementary heterothallic forms is unlimited; the number of true sexes is limited to two because there must be very close association of many adaptive features. All the above issues have, of course, been considered by many writers and especially clearly by Darlington ${ }^{36}$. Beadle's ${ }^{35}$ scheme has similarities but also fundamental differences; he, further, accepts Darlington's conclusion that nuclear fusion and meiosis must have arisen simultaneously, which is not necessary in the above scheme. Our contribution, regardless of hypothetical significance, is the experimental production of the third, and previously most inexplicable, step, and from Stern's ${ }^{5}$ work a suggested experimental technique for the production of fusion within a single organism. Our scheme has one advantage over others in that somatic reduction may in some cases (cf. ref. 22) have immediate selective value for the tissue involved and not only for the progeny ${ }^{36}$.

To conclude, the occurrence of polysomaty and polyteny in differentiated tissues and of somatic reduction divisions equivalent to both complete and incomplete meiosis are established, and also the optical doubleness (if not the multi-strandedness) of the chromatid which is nevertheless the unit of Mendelian segregation. The speculations on the possible significance of the data are predominantly of the nature of working hypotheses which can, though to very varying degrees, be tested. Their validity, or lack of it, at this stage is therefore immaterial to their possible usefulness. Further obvious speculations on issues such as the relationship of the postulated platelets to 'plasmagenes', etc., are not indulged in since we see at present no way for the cytogeneticist to test them.

It must be emphasized that though I accept full and sole responsibility for all statements above, the work is that of a team in which I have the collaboration of three senior and seven junior associates. In the detailed publications to follow, authorship and accrediting will indicate the particular contributions of each in so far as this is possible with a team the members of which, to end on a cytological analogy, are, like cells, individual units at the level of differentiation, that is, in their special studies, and sub-units at the integrative level, that is, in the general programme. Financial support is provided by the Wisconsin Alumni Research Foundation and, through the award of one fellowship, by the National Research Council, Committee on Growth.

\footnotetext{
${ }^{1}$ Schrader, F., Presidential Address, Section F, Amer, Assoc. Adv. Sci.

(1947).
"Darlington, C. D., "Recent Advances in Cytology" (Churchill, 1937).

'Huskins, C. L., J. Hered., 35, 81 (1944); 86, 43 (1946).

'Stern, H., Trans. Roy. Soc. Canada, 40, 141 (1946).

'Stern, H., J. Hered., 37, 46 (1946).

- Glaser, O., Trans. New York Acad. Sei., 4, 5 (1941).

? Sinnott, E. W., and Bloch, R., Amer. J. Bot., 33, 587 (1946).

${ }^{8}$ Geitler, L., Ber. Deutsch Bot. Ges., 58, 131 (1940).

- Lauber, Henriette, Ost. Bot. Z., 94, 30 (1947).

${ }^{10}$ Jăhnl, Gertrud, Chromosoma, 3, 48 (1947).

${ }^{11}$ Huskins, C. L., Proc. Roy. Soc. Canada (1947).

12 White, M. J. D., "Animal Cytology and Evolution" (Cambridge Univ. Press, 1945).

1s Berger, C. A., Pub. Carnegie Inst., Washington, 496 (1938).

" Huskins, C. L. (Discussion Cold Spring Harbor Symposium, June 1947 , in the press).

${ }^{15}$ Caspersson, T., Arch. Exp. Zellf., 22, 655 (1939).

14 Caspersson, T., Chromosoma, 1, 147 (1939).

${ }^{17}$ Gulick, Addison, Bot. Rev., 7, 433 (1941) (and personal conversation).

${ }^{13}$ Sharp, L. W., "Introduction to Cytology" (McGraw-Hill Book Co., 1934).

${ }^{19} \mathrm{Nebel,} \mathrm{B.} \mathrm{R.,} \mathrm{Z.} \mathrm{Zellf.} \mathrm{Mikr.} \mathrm{Anat.,} \mathrm{16,} 251$ (1932).

20 Kaufmann, B. P., Amer. J. Bot., 13, 59 (1926); Bot. Rev., in the press.
}

${ }^{21}$ Kodani, M. (in the press).

${ }^{28}$ Brown, Meta A., Amer. J. Bot., 34, 384 (1947).

${ }^{28}$ Stern, Curt, Genetics, 21, 625 (1936).

${ }^{24}$ Lederberg, J., and Tatum, E. L., Nature, 158, 558 (1946).

${ }^{25}$ Greenstein, J. R., "Biochemistry of Cancer" (Acad. Press. N.Y., 1947).

${ }^{26}$ Potter, V. R., IVth Int. Symp. on Cancer (in the press, 1947).

${ }^{27}$ East, E. M., Genetics, 19, 167 (1934).

${ }^{28}$ Nishiyama, I., Cytologia, 5, 146 (1933).

${ }^{20}$ Rousseau, J., Ecol. Soc. America, Amer. Assoc. Adv. Scl. meeting (1947).

${ }^{30}$ Muller, H. J., Proc. Roy. Soc., B, 134, 1 (1947).

si Wright, Sewall, Amer. Nat., 79, 289 (1945).

${ }^{32}$ Duncan, R. E., Amer. J. Bot., 32, 506 (1945).

${ }^{33}$ Goldschmidt, R., and Teh Ping Lin, Science, 105, 619 (1947).

34 Subramaniam, M. K., Curr. Sci., 16, 157, 228 (1947).

${ }^{85}$ Beadle, G. W., and Coonradt, Verna L., Genetic8, 29, 291 (1944).

"Darlington, C. D." “The Evolution of Genetic Systems" (Cambridge Univ. Press, 1939)

\section{THEORY OF ELECTROKINETIC EFFECTS}

\section{By F. BOOTH \\ H. H. Wills Physical Laboratory, University of Bristol}

$T$

THE purpose of this article is to summarize the present position of the theory of electrokinetic phenomena and to present certain new mathematical results I have obtained, the details of which will be published later.

In electrokinetic phenomena we are concerned always with the electrical double layer at the boundary between an electrolyte and a solid. Two types of theoretical problem arise in considering the effects of this double layer, depending upon whether equilibrium, or non-equilibrium irreversible conditions (in the thermodynamic sense), prevail. An example of the first type of problem is the determination of the mechanical force between two solid particles at rest immersed in an electrolyte also at rest. This problem, of fundamental importance in colloid chemistry, has been solved successfully by Verwey ${ }^{1}$ after many abortive attempts by other workers. The second type constitutes the subject matter of this article; here dynamic rather than static conditions prevail, and we have to determine various consequences of the effect of the double layer on the flow of an electrolyte past a solid surface. These electrokinetic effects have, apart from their intrinsic interest, important industrial and biological applications ${ }^{2}$. The main electrokinetic phenomena are:

1. Cataphoresis or electrophoresis; this is the motion of the particles in a suspension when an electric field is applied (Reuss, 1808).

2. Electro-osmosis; this is the flow of a liquid through a capillary when an electric field is applied parallel to its axis. Closely related is the effect known as electro-osmotic pressure; if the flow through a horizontal capillary is prevented, a difference of pressure develops between the ends when the field is applied (R. Porret, 1816).

3. Streaming potentials; the potential set up between the ends of a capillary when an electrolyte is forced through it (Quincke, 1859).

4. Sedimentation potentials. A suspension of particles, if allowed to settle, sets up a vertical electric field (Dorn, 1878). A very similar effect is the difference of potential which develops between the nodes and antinodes set up by stationary sound waves in a suspension (see, for example, Hermans ${ }^{8}$ ). 
5. Electroviscous effect; this is the increase of the effective viscosity of a solid suspension owing to the double layer on the particles.

Other effects may be suggested ; for example, the double layer must influence the rate of diffusion of particles in suspension.

The classical theory of effects (1) to (4) is due to Helmholtz ${ }^{4}$ and Smoluchowski ${ }^{5}$. For electro-osmosis they gave, for the mean velocity of flow,

$$
\bar{v}=\frac{E \varepsilon \zeta}{4 \pi \eta} .
$$

Here $E$ is the field-strength, $\varepsilon$ the dielectric constant of the liquid, $\zeta$ the potential difference across the double layer, $\eta$ the coefficient of viscosity. Smoluchowski's contribution was to insert $\varepsilon$ in (1) and to show that the assumption for the capillary, of a uniform cross-section normal to $E$, could be discarded. Thus (1) holds for a closely packed powder with electrolyte filling the interstices. It is thus easily seen that the velocity $U$ of cataphoresis will also be given by (1), $U$ replacing $\bar{v}$, and $\zeta$ now being the electrokinetic potential of the particles. For, suppose we have a fixed but isolated particle; at a large distance the mean streaming velocity will be given by (1). But this system is equivalent to a stationary liquid and a moving particle.

The field set up in streaming potentials is

$$
E=\frac{\varepsilon p \zeta}{4 \pi \eta \sigma}
$$

where $p$ is the pressure gradient and $\sigma$ the specific conductivity of the electrolyte. By an argument very similar to that we used to relate $U$ to $\bar{v}$, equation (2) can be used to find the field set up in sedimentation. This is

$$
E=\frac{1}{3} \frac{\varepsilon \zeta g\left(\Delta-\Delta^{\prime}\right)}{\pi \sigma \eta}, .
$$

$\Delta$ being the particle density and $\Delta^{\prime}$ the fluid density.

These formulæ only apply provided that the thickness of the double layer at each point of the interface is small compared with the radius of curvature at that point.

Although the Helmheltz-Smoluchowski formulæ have been extensively used for the analysis of experiments, they have undergone considerable criticism and modification in recent years. Let us first examine the phenomenon of cataphoresis; physically, this is the simplest effect but it is the most difficult one to treat mathematically. One obvious limitation of the Helmholtz-Smoluchowski theory is the restriction on the thickness of the double layer. This is not likely to be serious in effects (2) and (3), but evidently may not apply in cases (1), (4) and (5) for very small particles. For cataphoresis, Henry ${ }^{6}$ obtained a formula which did not require this restriction on the thickness of the double layer. For non-conducting spherical particles he obtained

$$
\begin{array}{r}
U=\frac{\varepsilon E \zeta}{6 \pi \eta}\left[1+\frac{1}{16} b^{2}-\frac{5}{48} b^{3}-\frac{1}{96} b^{4}+\frac{1}{96} b^{5}+\right. \\
\left.\frac{1}{8} b^{4} e^{b} E_{i}(b)\left(1-\frac{b^{2}}{12}\right)\right], . .
\end{array}
$$

where $E_{i}(x)=\int_{x}^{\infty} \frac{e^{-u}}{u} d u, b=\chi a, a$ is the particle radius, and

$$
\chi^{2}=\frac{4-e^{2}}{\varepsilon k T} \sum_{i=1}^{8} n_{i} z_{i}{ }^{2} .
$$

The summation is over the $s$ ionic species present in the electrolyte, $n_{i}$ being the concentration, $z_{i}$ the valency. Since $\chi^{-1}$ is a measure of the double layer thickness, $b$ represents the ratio of the radius $a$ to this thickness. The function in brackets tends to $3 / 2$ for $b$ large, and to 1 for $b$ small; hence in the limit (3) agrees with Smoluchowski's formula.

Henry's treatment, however, contains a serious blemish, for in order to obtain (3), he had to assume that the charge distributions on the surface and in the electrolyte remain spherically symmetrical when the field is applied; this is certainly incorrect, since the ions will tend to fall behind the particle, causing excess of charge there and a deficit in front, as compared with the equilibrium charge density. This behaviour is known as the 'relaxation effect' in the theory of electrolytes.

Various attempts have been made to remedy this defect ; one by Hermans ${ }^{7}$ and another by Komagata ${ }^{8}$ are valueless since they contain fundamental mistakes. More recently, Overbeek ${ }^{9}$ and, independently, the present writer ${ }^{10}$, have given generalizations of Henry's formula. I find a general expression for $U$ in the form

$$
\begin{array}{r}
U=\frac{E e Q}{6 \pi \eta a} X_{1}(b)+\frac{E}{6 \pi \eta} \stackrel{\infty}{r=2}_{r}^{\infty} Q^{r} e^{2 r-1} a^{-r}(\varepsilon k T)^{1-r} \\
{\left[q_{r} X_{r}(b)+Y_{r}\left(b, q_{\mu}, t\right)+Z_{r}\left(b, q_{\mu} q_{\mu}^{*}, t\right)\right\rceil .}
\end{array}
$$

Qe denotes the total charge on the particle when at rest, $q_{\mu}$ the set $\left(q_{2}, \ldots q_{r}\right), q_{\mu}{ }^{*}$ the set $\left(q_{3}{ }^{*}, \ldots q_{r}{ }^{*}\right)$; also

$$
\begin{aligned}
& q_{r}=\sum_{i=1}^{8} n_{i} z_{i}^{r+1} / \sum_{i=1}^{8} n_{i} z_{i}^{2} \text {, and } \\
& q_{r}{ }^{*}=\varepsilon k T \sum_{i=1}^{8} n_{i} z_{i}^{r-1} w_{i}^{-1} / \pi \eta e^{2} \sum_{i=1}^{8} n_{i} z_{i}{ }^{2},
\end{aligned}
$$

where $w_{i}$ is the ionic mobility of the ion of type $i$. The functions $X_{r}, Y_{r}$ and $Z_{r}$ are very complicated, although it can be shown that they all, except $X_{1}$, tend to zero as $b$ tends to zero or to infinity; the $X_{r}$ are functions of $b$ only, but $Y_{r}$ and $Z_{r}$ may contain certain surface parameters which we have indicated by $t$ in (4). The physical significance of the three terms in the square brackets is as follows: $X_{r}$ represents a contribution to $U$ due to the symmetrical part of the field around the particle. These terms, when $r$ is greater than 1, do not appear in Henry's formula (which is identical with the first term of (4)) because he adopted the Debye-Hückel approximation, namely, that $\zeta_{e} / k T \ll 1 ; \zeta_{e} / k T$ may reach values of about 4 , so that this approxi. mation is not good. $Y_{r}$ represents a contribution to $U$ due to the distortion of the field, but not involving the ionic mobilities; that is, distortion not due to relaxation ; $Z_{r}$ is the contribution due to relaxation. So far, the expansion has been worked out as far as the $Q^{4}$ terms for symmetrical electrolytes (that is, electrolytes with equal numbers of positive and negative ions, or with $q_{2 r}=0$ ), though work is proceeding on the more difficult case of unsymmetrical electrolytes.

To obtain formula (4) it is first necessary to derive the spherically symmetrical part of the field. At distance $r$ from the centre, the potential of this field is found to be

$\psi_{1}=\sum_{m=1}^{\infty} Q^{m} e^{2 m-1}(\varepsilon a)^{-m}(-k T)^{1-m} \lambda_{m}(\chi r) .$.

The functions $\lambda$ are obtained by transforming the differential equation for the potential into an integral 
equation. By analogy with (5) we write for the distortion of the potential of the ionic atmosphere

$$
\begin{aligned}
& \varphi(r, \theta)=E \sum_{\nu=1}^{\infty} \sum_{n=0}^{\infty} Q^{v} e^{2 v}(\varepsilon k T)^{-v} \\
& a^{1-v} \lambda_{v, n}(r) P_{n}(\cos \theta),
\end{aligned}
$$

$\theta$ being the angle co-ordinate. Provided $\psi_{1}$ and $\varphi$ are known, $U$ can be calculated. Hence the problem is resolved into the determination of the functions $\lambda_{y, n}$. They are found from the equations of motion of the electrolyte and of the ions.

A serious difficulty for any theory of cataphoresis is to obtain satisfactory boundary conditions for the solid-liquid interface. We have no exact knowledge of the structure of the interface, and indeed it is from experiments like electrophoresis that we must derive information. Hence it is very desirable, if possible, to formulate basic assumptions on the surface state, in as broad a manner as possible, precluding specific mechanisms. It has been possible to derive (4) with the following simple assumptions about the interface :

(I) The thickness of the region in which the surface charge resides is small compared with the particle radius.

(II) The charge in this surface region is immobile.

(III) The surface charge density at any point is an explicit function of the ionic concentrations in the adjacent electrolyte and of the temperature only. (The surface parameters in (4) are partial derivatives of this function with respect to $n_{i}$.)

Assumption I is almost certainly true; II may be incorrect, but surface conductance is unimportant provided $\sigma_{s} \ll \sigma a$ (where $\sigma_{s}$ is the surface conductance). Assumption III is more speculative but it seems very plausible; for example, in conjunction with assumption II, it would certainly be valid for a surface charge produced by adsorption of ions.

Overbeek expresses $U$ in a series of powers of $\zeta$ (up to the third power). Using (5) to relate $Q$ and $\zeta$, his results should give formula (4) correct to $Q^{3}$; agreement seems fairly satisfactory although his method is rather different from ours. He employs, however, a more restrictive assumption than III above, namely, that the charge density on the surface is fixed; this cannot be strictly correct. Another basic assumption made by Henry is that the 'inertia terms' in the equations of motion of the electrolyte tan be dropped. It can be shown that this is equivalent to assuming that $U$ is proportional to the field strength $E-$ a result often experimentally confirmed. A more exact formula for $U^{11}$, taking into account the inertia terms, is

$U=\frac{E e Q}{6 \pi \eta a} X_{1}(b)+\sum_{r=2}^{\infty}(E e Q)^{r} \eta^{-2 r}\left[W_{r}(b)+O(Q)\right]$.

The series of functions $W_{r}$, of which the first two members have been found, are, like $X_{r}, Y_{r}$ and $Z_{r}$, very complicated. Oseen's linearized equations were used to deduce (7) ; in fact, the problem is closely related to a well-known problem of hydrodynamics - the determination of the drag on a sphere moving through a liquid. The most complete solution of this problem is due to Goldstein ${ }^{12}$, and we have used his analysis extensively.

Another improvement of Henry's formula is to take into account the effect of surface conductance. For an insulating particle with a surface conductance $\sigma_{\delta}$, and with a thin double layer, we find ${ }^{13}$ that the right-hand side of (3) should be multiplied by the factor $\sigma a\left(\sigma_{s}+\sigma a\right)^{-1}$, where $\sigma$ is the ordinary bulk conductance of the electrolyte. The importance of the surface conductance effect evidently depends upon the relative magnitudes of $\sigma$ and $\sigma_{8}$. Some experiments by Rutgers ${ }^{14}$ suggest that the interface may possess very high surface conductance; hence surface conductance may be of great importance in interpreting the results of cataphoresis measurements. It is natural to inquire if a formula of type (4) might be given in this case. There are no additional mathematical difficulties, but unfortunately we require much more detailed assumptions than I-III for the conditions at the surface. For example, we should require the rate of 'condensation' of ions on to the surface and the rate of 'evaporation'.

Another problem under consideration is the determination of the rate of electrophoresis for solid particles in the form of ellipsoids. This should be of use in biological applications where spherical particles are not always available, for example, in the investigation of proteins.

We shall now consider the sedimentation potential. Smoluchowski's formula for the sedimentation potential, like his formula for electrophoresis, applies only to a thin double layer. I have extended this to the general case ${ }^{15}$ by using methods similar to those for formula (4). As is to be expected, the removal of the condition $b \gg 1$ introduces a complicated function of $b$ and of the surface parameters on the right-hand side of equation (3).

Finally, we come to the electroviscous effect. The first calculation of the effective viscosity $\eta$ of a solid suspension of spheres in a liquid of viscosity $\eta_{0}$ was made by Einstein ${ }^{16}$, who found

$$
\eta=\eta_{0}\left(1+\frac{5}{2} \frac{v}{V}\right), . . . .
$$

where $v$ is the volume of solid, $V$ of the suspension. This formula only applies for $v \ll V$. A better formula was given by Guth", who added the term $109 v^{2} / 14 V^{2}$. Smoluchowski ${ }^{18}$ in 1916 pointed out that a charge on the solid particles should increase the viscosity, and published without proof the following formula :

$$
\eta=\eta_{0}\left(1+\frac{5}{2} \frac{v}{V}\left[1+\frac{1}{\sigma \eta_{0} a^{2}}\left(\frac{\zeta \varepsilon}{2 \pi}\right)^{2}\right]\right) .
$$

Later, Krasny-Ergen ${ }^{19}$ gave a proof ; his equation being identical with (9) except for the multiplication of the last term by $1 \cdot 5$. Both formulæ only apply for a thin double layer. I have, however, succeeded in finding a formula for $\eta$, without this restriction ${ }^{20}$. The more general formula does not agree with equation (9), and I believe that Krasny-Ergen's treatment is incorrect. With this, we conclude our account of recent theoretical developments.

Much further work remains to be done, but at this stage it would seem that a thorough experimental check of theory would be more valuable than continued elaborations of the latter. The most useful procedure would be the determination of the electro. kinetic potential $\zeta$ for a given surface and electrolyte by as many different methods as possible. The results would indicate if the theory required drastic or only minor modifications. In reading the literature, the impression is inescapable that in the past much effort has been wasted because experimentalists have confidently used formulæ without realizing their limitations, or at any rate without attempting to ascertain, so far as possible, whether basic assumptions for their validity were satisfied in their work. 
Only when a theory is well tested can it be applied to new experimental data and conclusions drawn therefrom with a reasonable degree of certainty.

1 Verwey, Philips Laboratory Research Reports, 1, 33 (1945).

"The Flectrical Double-Layer : a Symposium", Trans. Farad. Soc., 36 , pt. 1, 1 (1940).

3 Hermans, Phil. Mag., 26, 674 (1938).

- Helmholtz, Ann. der Phys., 7, 337 (1879).

- Smoluchowski, "Graetz' Handbook", 2, 374 (1921).

' Henry, Proc. Roy. Soc., A, 133, 106 (1931).

'Hermans, Phil. Mag., 26, 650 (1938).

Komagata, Researches of the Electrotechnical Laboratory of Tokyo, No. 387 (1935).

Overbeek: Sonderabdruck a. d. Kolloid-Beiheften, 54, 287 (1943).

107

$12\}$

Booth, to be published shortly in Proc. Roy. Soc. and other journals.

20

12 Goldstein, Proc. Roy. Soc., A, 123, 225 (1929).

14 Rutgers, Trans. Farad. Soc., 36, 69 (1940).

16 Einstein, Ann. der Phys., (4), 19, 289 (1906).

17 Guth, Koll. Z., 74, 147 (1936).

10 Smoluchowski, Koll. Z., 18, 194 (1916).

to Krasny-Frgen, Koll. Z., "74, 172 (1936).

\section{OBITUARIES}

\section{Dr. Charles Bolton, F.R.S.}

ThE death on December 6 of Charles Bolton, at the age of seventy-seven years, removes one more member of that distinguished band of clinicians who, during the opening years of the century, so successfully combined general consulting practice and fundamental research. The impulse to contribute to the knowledge of his subject was undoubtedly the result of his early contacts. When Bolton went to University College, London, the Medical School had not yet separated from its parent body, and the department at University College combined both clinical and pre-clinical subjects and housed all the laboratories. At that time the medical faculty comprised a galaxy of talent. Thane and Schafer were at the height of their powers, and were soon to be joined by Bayliss and Starling. On the clinical side were Ringer, Barlow, Rose Bradford, Victor Horsley and Sidney Martin. Coming into this atmosphere as he did, fresh from an apprenticeship in general practice, Bolton was, as he often told later, first astonished and bewildered, and then inspired. He never lost the scientific enthusiasm then aroused. Following medical qualification, he began investigations on the bacteriology of typhoid fever and the morbid anatomy of diphtheria. These attracted sufficient attention to gain for him, in 1903, the Grocers' Company research studentship, and so the opportunity for laboratory research. This he utilized so well that five years later he was appointed, on a part-time basis, first director of the new Graham Research Laboratories at his own Medical School. This post he held until 1914, when it was merged with the lectureship in pathology-which Bolton also held-in a single whole-time post to which the late Prof. A. E. Boycott was appointed. But Bolton continued to work part-time in the Graham Department until the outbreak of another war, in 1939, closed his Medical School and so brought to an end his researches. In the intervening quarter of a century Bolton had been a busy general consulting physician, in wide demand, and with no source of livelihood but his practice. Yet, throughout that time, at least one afternoon a week was reserved for research.
Bolton will be remembered chiefly for his work on two subjects : the formation of tissue fluids and œdema, and the production of peptic ulceration. His interest in odema and ascites sprang directly from clinical observations and particularly those on a case of illness which, nowadays, would be diagnosed as constrictive pericarditis. At this time the teaching of his friend Ernest Starling, that the rate of formation of tissue fluid was determined by the balance of hydrostatic and osmotic pressures across the capillary membrane, was gaining acceptance. Bolton set. himself to determine the factors concerned in ascites. Re-reading his work to-day, it is impressive to note how often Bolton's conclusions, once, regarded as strange, have been vindicated by subsequent discoveries. By direct experiment he showed that increased venous pressure in itself could not account for the ascites, and he clearly stated : "The abnormal output of lymph leading to dropsy is primarily conditioned by the nutritive state of the capillary wall". Thirty years later, Landis reached the same conclusions from his demonstration of the deleterious effect of anoxia on capillary permeability. Again, the increased blood volume in cardiac failure had been discovered by Lorrain-Smith, and was then considered to be one of the causes of dropsy. Bolton grasped clearly that, under such abnormal conditions, fluid may be lost into one part of the body so that the remainder becomes dehydrated. He wrote: "The increase in blood volume must, therefore, be looked upon as a secondary phenomenon and due to an effort of the animal to make up its normal quantity of blood in the anæmic areas, and to replace the fluid lost as dropsy". Modern measurements of blood volume, cardiac output and tissue-fluid spaces would essentially confirm this conclusion, which seemed so strange when first enunciated.

Bolton's main contribution to our knowledge of peptic ulceration concerned the conditions necessary to produce acute ulceration. By injecting suspensions of one animal's gastric mucosa into another species, he was able to produce an anti-serum which, on intraperitoneal injection into the donor species, caused acute ulceration in the stomach. The interesting point then emerged that the gastro-toxic serum produced no visible effect on the gastric mucosa unless this were in contact with acid gastric juice. Neutralize the acid and no ulcers occurred ; angment it directly, or by suitable feeding, and ulceration was increased. Clearly the gastrotoxin had produced a definite, if invisible, temporary damage to the gastric mucosa which deprived it of its natural resistance to acid. Bolton believed that such damage occurred in the course of disease, and so led to acute ulceration. With Cruveilhier he believed that chronic peptic ulcers originated as acute ulcers; but although he tried repeatedly, he could not prevent the healing of the acute ulcers he produced by gastrotoxins. By artificially delaying the emptying of the stomach, by augmenting acidity, he succeeded in delaying, but not in preventing, healing. It now seems probable that, if he had only maintained a consistent hyperacidity, he might have seen his acute ulcers become chronic. Nevertheless, he had clearly appreciated the importance of acidity in exaggerating and delaying the healing of gastric lesions ; and it was on that basis that he formed his therapy of these illnesses.

Personally, Bolton was a massive Yorkshireman with a typical dry humour. He possessed a remarkable power of effortless concentration which rendered him cheerfully impervious to all distractions. Yet he was 